\title{
THE NEUTRAL PION YIELD IN THE STATISTICAL MODEL FOR $\mathrm{e}^{+} \mathrm{e}^{-}$ANNIHILATION
}

\author{
J. ENGELS and K. FABRICIUS \\ Department of Theoretical Physics, University of Bielefeld, Germany
}

K. SCHILLING

Physics Department, University of Wuppertal, Germany

Received 20 August 1974

\begin{abstract}
We consider the decay of neutral fireballs with mass $M$ into $\pi^{+,-, o}$ and derive the first two leading terms of the average multiplicities as a function of $\boldsymbol{M}$. We discuss the implication of this result for a statistical bootstrap description of $\mathrm{e}^{+} \mathrm{e}^{-}$annihilation into hadrons.
\end{abstract}

It has been proposed, that the virtual photon in $\mathrm{e}^{+} \mathrm{e}^{-}$annihilation into hadrons has the properties of a fireball in the sense of Hagedorn [1] and that therefore this process should be the proper reaction to study fireballs in some detail [2]. Indeed the preliminary results at SLAC support this picture insofar as the mean energy of secondaries measured in $\mathrm{e}^{+} \mathrm{e}^{-}$hadrons is small, as predicted by this model. There has been some theoretical effort to incorporate isospin conservation into the statistical bootstrap scheme for nonexotic fireballs, in order to facilitate predictions on charged secondaries. The linear bootstrap problem has been completely solved [3-5], whereas in the case of the full statistical bootstrap (with isospin phase space weights) only the fireball level density and total pion multiplicity distribution have been investigated [5]. In view of the preliminary experimental result, that the mean total energy of charged secondaries in $\mathrm{e}^{+} \mathrm{e}^{-} \rightarrow$ hadrons, is only about $50 \%$ of the incoming energy [6], one is confronted to the question, whether a statistical approach can possibly account for a sizeable deviation from $\left\langle E_{\mathrm{ch}}\right\rangle=2\left\langle E_{\text {neutral }}\right\rangle$. This has been answered to the negative in the framework of the linear bootstrap with only pions as input.

This raises the question, whether such a deviation can be explained at all in the framework of a bootstrap scheme. In this context it seems appropriate to start from a model for isovector fireballs only and pions as input particles:

$$
\begin{aligned}
2 Z_{m}=t_{m}+\sum_{\substack{N_{+}, N_{0}, N_{-} \\
\Sigma N_{i}>1, N_{+}-N_{-}=m}}^{N_{\max }} P^{(1)}\left(N_{+}, N_{\mathrm{o}}, N_{-}\right) \\
\times Z_{+}^{N_{+}} Z_{\mathrm{o}}^{N_{\mathrm{o}}} Z_{-}^{N_{-}},
\end{aligned}
$$

where $Z_{m}=\mathcal{L}\left(B_{m} \tau_{m}\right)=B_{m} \int \mathrm{d}^{4} q \tau_{m}\left(q^{2}\right) \exp (-\beta q)$, with $\beta_{\mu}=(\beta, 0,0,0)$ is the Laplace-transform of the fireball states with isospin $I=1, I_{3}=m$, and $P^{(1)}\left(N_{+}, N_{-}, N_{\mathrm{o}}\right)$ are Cerulus coefficients [7]. Furthermore, $t_{m}=\mathscr{L}\left(B_{m} \Theta\left(q_{0}\right) \delta\left(q^{2}-m_{\pi}^{2}\right)\right)$. In the full bootstrap scheme, $N_{\max }=\infty$. The singularity structure of $Z_{m}$ yields the asymptotic expansion

$\left\langle N_{i}\right\rangle=a_{1}^{(i)} M+a_{0}^{(i)}+a_{-1}^{(i)} M^{-1}+\ldots$

in terms of the fireball mass $M$. In this note we calculate the coefficients $a_{1}^{(i)}, a_{0}^{(i)}$ for the decay of a neutral fireball.

The general expression for $\left\langle N_{i}\right\rangle$ reads simply

$\left\langle N_{i}\right\rangle=\frac{\mathcal{L}^{-1}\left(t_{i} \partial Z_{0} / \partial t_{i}\right)}{\mathcal{L}^{-1}\left(Z_{0}\right)}$.

The model given by eq. (1) leads to a square root type singularity for $Z$, i.e.

$$
\begin{aligned}
& Z_{0}=z_{1 / 2}\left(t_{\mathrm{s}}-t\right)^{1 / 2}+z_{3 / 2}\left(t_{\mathrm{s}}-t\right)^{3 / 2}+\ldots \\
& \quad+\text { terms regular at } t=t_{\mathrm{s}}, \\
& t_{i} \frac{\partial Z_{0}}{\partial t_{i}}=d_{-1 / 2}^{(i)}\left(t_{\mathrm{s}}-t\right)^{-1 / 2}+d_{1 / 2}^{(i)}\left(t_{\mathrm{s}}-t\right)^{1 / 2}+\ldots
\end{aligned}
$$




$$
+ \text { terms regular at } t=t_{\mathrm{s}} .
$$

After a lengthy calculation eqs. (3.4) lead to the result

$\left\langle N_{\mathrm{ch}}\right\rangle=2 \sigma M / m_{\pi}-3 \sigma^{2}+2 B_{+}$

$\left\langle N_{0}\right\rangle=\sigma M / m_{\pi}-\frac{3}{2} \sigma^{2}+B_{0}$

with

$B_{i}=-\frac{t_{\mathrm{s}}}{6}\left(\frac{d_{1 / 2}^{(i)}}{d_{-1 / 2}^{(i)}}-3 \frac{z_{3 / 2}}{z_{1 / 2}}\right) ; \quad \sigma=\frac{1}{3} \frac{K_{1}\left(m_{\pi} \beta_{0}\right)}{K_{2}\left(m_{\pi} \beta_{0}\right)}$

$\beta_{0}^{-1}=T_{0}=$ Hagedorn temperature.

The quantities $z_{1 / 2}, \ldots, d_{1 / 2}$ can be evaluated in form of power series by inserting the eqs. $(4 a, 4 b)$ into the bootstrap equation and its appropriate derivatives

with respect to $t_{i}$.

For comparison we list the asymptotic expressions obtained in the linear bootstrap

$\left\langle N_{\mathrm{ch}}\right\rangle=2 \sigma M / m_{\pi}+6 \sigma^{2}+9 \sigma / m_{\pi} \beta_{0}-\frac{4}{9}$

$\left\langle N_{0}\right\rangle=\sigma M / m_{\pi}+3 \sigma^{2}+\frac{9}{2} \sigma / m_{\pi} \beta_{0}-\frac{5}{9}$.

As is well known, the leading terms are independent of the charge observed and depend solely on the Hagedorn temperature $T_{0}=1 / \beta_{0}$. The next to leading order terms in eq. (5) appear as a sum of two terms: $\sigma$ depends only on $T_{0}$ and not on $N_{\max }$, whereas $B_{+, 0}$ are independent of $T_{0}$. Moreover, the difference $\left\langle N_{+}\right\rangle-$ $\left\langle N_{0}\right\rangle$ does not depend on $T_{0}$, but only on the degree $N_{\max }$ of the bootstrap. We have evaluated the quantities $B$ for $2 \leqslant N_{\max } \leqslant 45$. As one can see from table 1 , the $B$ 's converge rapidly to their full bootstrap limits.

Table 1

\begin{tabular}{rll}
\hline$N_{\max }$ & $B_{0}$ & $B_{+}$ \\
\hline 2 & $9.26 \times 10^{-2}$ & $2.03 \times 10^{-1}$ \\
3 & $1.41 \times 10^{-1}$ & $1.80 \times 10^{-1}$ \\
4 & $1.33 \times 10^{-1}$ & $1.83 \times 10^{-1}$ \\
5 & $1.34 \times 10^{-1}$ & $1.83 \times 10^{-1}$ \\
45 & $1.34 \times 10^{-1}$ & $1.83 \times 10^{-1}$ \\
\hline
\end{tabular}

The values given in this table lead to the result that $\left\langle N_{0}\right\rangle\left\langle\left\langle N_{\mathrm{ch}}\right\rangle\right.$ for $M \geqslant 3 \mathrm{GeV}$ is bounded by

$$
0.49 \leqslant\left\langle N_{0}\right\rangle /\left(N_{\text {ch }}\right\rangle \leqslant 0.5 \quad \text { (at } T_{0}=160 \mathrm{MeV} \text { ) }
$$

and therefore very close to its asymptotic value.

Since the average energy of secondaries is independent of their charge, we thus come to the conclusion that the full bootstrap model with input pions cannot account for the experimentally observed deviation from $\left\langle E_{\mathrm{ch}}\right\rangle=2\left\langle E_{\text {neutral }}\right\rangle$. This result does not change, if in this framework other input particles that may strongly decay into pions are introduced.

Therefore, unless one is willing to change the basic assumptions of the statistical bootstrap model, one is forced to allow the production of particles whose decay violates isospin conservation, such as $\eta$. A priori, the bootstrap model does not restrict the $\eta$ production rate. A value of $\left\langle N_{\pi \mathrm{ch}}\right\rangle /\left\langle N_{\pi}\right\rangle=0.55$ would require a ratio $\left\langle N_{\eta}\right\rangle /\left\langle N_{\pi^{0}}\right\rangle=0.5$. An $\eta$ yield of this surprisingly large order has recently been observed by Caldwell et al. [8] in $\gamma_{\mathrm{p}}$ collisions at $p_{\mathrm{T}} \geq 1 \mathrm{GeV} / c$. It remains to be seen that $\eta$ 's are indeed produced so abundantly in $\mathrm{e}^{+} \mathrm{e}^{-}$annihilation.

\section{References}

[1] R. Hagedorn, CERN preprint 71-12;

S. Frautschi, Phys. Rev. D3 (1971) 2821.

[2] J. Engels, H. Satz and K. Schilling, Nuovo Cimento 17A (1973) 535.

[3] J. Kripfganz and E.M. Ilgenfritz, Physics Letters 48B (1974) 329;

The result, eq. (16), of this paper is wrong. For the correct form, see ref. [5] eq. (D. 10).

[4] F. Csikor, I. Farkas, Z. Katona and I. Montvay, Nuclear Physics B4 (1974) 343.

[5] J. Engels, K. Fabricius and K. Schilling, Nuovo Cimento $A$, in press.

[6] B. Richter, rapporteur's talk given at the 17th Intern. Conf. on High energy physics, London, July 1974.

[7] F. Cerulus, Nuovo Cimento 19 (1960) 528.

[8] D.O. Caldwell, Contribution 302 to the 17 th Intern. Conf. on High energy physics, London, July 1974. 